\title{
El Modelo Piramidal: alternativa feminista para analizar la violencia contra las mujeres
}

\author{
Esperanza Bosch-Fiol' (iD 0000-0002-0635-6847 \\ Victoria Aurora Ferrer-Perez' (DD 0000-0002-8096-4031 \\ 'Universidad de las Islas Baleares, Departamento de Psicología, Palma de Mallorca, ES/ \\ Baleares, España. 07122
}

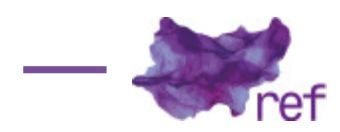

Resumen: El objetivo de este trabajo es presentar una propuesta de modelo explicativo para la violencia contra las mujeres llamado modelo Piramidal que pretende responder a dos objetivos: (a) Recoger los principales elementos incluidos en diferentes modelos explicativos multicausales, completando algunos de sus aspectos. Particularmente, profundiza en analizar cómo se conectan entre sí los factores explicativos de esta violencia y en aquellos mecanismos que permiten comprender porqué ciertos varones eligen ejercer violencia contra las mujeres. (b) Ser aplicable a las diferentes formas de violencia contra las mujeres, incidiendo en los elementos comunes a todas ellas. El modelo resultante, al que se ha denominado modelo piramidal, incluye cinco etapas o escalones (sustrato patriarcal, socialización diferencial, expectativas de control, factores desencadenantes y estallido de la violencia) y un proceso de filtraje.

Palabras clave: modelos; violencia; socialización; factores desencadenantes.

The Pyramid Model: A Feminist Alternative for Analysing Violence against Women

Abstract: The objective of this paper is to propose an explanatory model for violence against women called pyramidal model, that aims to respond to two objectives: First, this model includes the key elements that are already included in the available multi-causal explanatory models, supplementing some aspects of these models. In particular, it presents a deeper analysis of how to connect together the various factors behind this violence and those mechanisms that allow us to understand why some men choose to exercise violence against women. Secondly, this model has been developed with the intention to apply to different forms of violence against women, deepening the analysis of elements common to them all. The resulting model, which has been called pyramidal model, includes five stages or steps (the patriarchal substrate, differential socialization, expectations of control, triggers, and outbreak of violence) and a filtering process.

Key words: Models; Violence; Socialization; Triggers.

O modelo piramidal: uma alternativa feminista para analisar a violência contra as mulheres

Resumo: O objetivo deste trabalho é apresentar uma proposta de modelo explicativo para a violência contra as mulheres chamado modelo Piramidal, que pretende responder a dos objetivos: (a) Coletar os principais elementos incluídos em diferentes modelos explicativos multicausais, completando alguns de seus aspectos. Particularmente, nos aprofundamos em analisar como os fatores explicativos da violência estão interligados, e naqueles mecanismos que permitem compreender por que determinados homens escolhem exercer violência contra as mulheres. (b) Ser aplicável às diferentes formas de violência contra as mulheres, incidindo em todos os elementos comuns a todas elas. O modelo resultante, denominado modelo piramidal, inclui cinco etapas ou estágios (substrato patriarcal, socialização diferencial, expectativas de controle, fatores desencadeantes e surtos de violência) e um processo de filtragem.

Palavras-chave: modelos; violência; socialização; fatores desencadeantes. 


\section{Introducción}

En el análisis de la violencia contra las mujeres, y, particularmente, de la que ocurre en la pareja, se han manejado diferentes modelos explicativos que han ido evolucionando a lo largo del tiempo (Roberta ALENCAR-RODRIGUES; Leonor CANTERA, 2012; Esperanza BOSCH; Victoria FERRER, 2002; Macarena BLÁZQUEZ; Juan Manuel MORENO; M. Elena GARCÍA BAAMONDE, 2010; Kenneth CORVO; Ellen DELARA, 2010; Francisca EXPÓSITO; Miguel C. MOYA, 2005; Michele HARWAY, 2002; Jorge RODRÍGUEZ-MENÉS; Ana SAFRANOFF, 2012). Así, a partir de la década de 1960 comenzaron a formularse modelos unicausales que consideraban esta violencia como producto de la enfermedad o el desorden psicológico, y buscaban su causa en características individuales (biológicas, psicopatológicas y/o de personalidad), tanto del agresor como de la víctima. Posteriormente, se desarrollaron modelos más amplios y complejos, entre los que estarían modelos de tipo psicológico, como el del aprendizaje social, de los recursos, del intercambio, o de la transmisión intergeneracional de la violencia, por mencionar algunos; y también modelos de tipo sociológico. Entre estos últimos destacan especialmente (Jennifer LAWSON, 2012): la denominada perspectiva de la violencia o el conflicto familiar, que entiende esta violencia como una expresión del conflicto dentro de la familia, de modo que puede ser considerada como un producto cultural derivado de aquellos factores sociales organizacionales que contribuyen al uso de la violencia en general, y que no tienen una relación específica con el género; o la perspectiva teórica feminista que entiende, por el contrario, que la violencia que ocurre en la vida privada y en la pareja no es ajena al control social, y la considera como violencia basada en el género, como un producto de los valores culturales patriarcales, como un mecanismo de control social para mantener la subordinación de las mujeres respecto a los hombres.

Actualmente, y tomando en consideración el carácter complejo de la violencia contra las mujeres en sus diferentes formas (Lori L. HEISE, 1997), hay coincidencia en considerar que su análisis debe ser realizado desde modelos explicativos de tipo multicausal (BOSCH; FERRER, 2002; Teresa CABRUJA, 2004; HARWAY, 2002; HEISE, 1997; Jesús L. MEGÍAS; Pilar MONTAÑÉS, 2010; ONU, 2006; OPS, 2013; RODRÍGUEZ-MENÉS; SAFRANOFF, 2012). La característica común a estos modelos es que, aunque pueden diferir en la importancia que asignan a los diferentes factores individuales y sociales considerados, todos ellos entienden esta violencia como un fenómeno complejo, que sólo puede ser explicado a partir de la intervención de un conjunto de factores específicos, en el contexto general de las desigualdades de poder entre varones y mujeres, en los niveles individual, grupal, nacional y mundial (ONU, 2006). Es decir, estos modelos no sólo tomarían en consideración la existencia de múltiples factores que contribuyen a la génesis y/o mantenimiento de esta violencia, sino que consideran que el género y las relaciones de género desempeñan un papel clave en la violencia ejercida por los hombres hacia las mujeres (APA, 1999), e incorporan como clave explicativa la vinculación de esta violencia con una sociedad dicotomizada y estructurada desigualmente en función del género (CABRUJA, 2004; Carmen DELGADO, 2013; Concepción FERNÁNDEZ VILLANUEVA, 2004), si bien la importancia que otorgan a esta cuestión puede variar de unos modelos a otros.

Entre los modelos multicausales formulados específicamente para explicar la violencia contra las mujeres en el contexto de la pareja estarían, por ejemplo, los de autoras y autores como: Sandra Stith y Sarah Farley (1993); James O'Neil y Harway (1999); Enrique Echeburúa y Javier Fernández-Montalvo (1998); o Lori L. Heise (1998).

Rodríguez-Menés y Safranoff (2012), por su parte, diferencian cinco modelos formulados para explicar la violencia contra las mujeres en la pareja: Las perspectivas de la violencia familiar, el sexismo y la inconsistencia de estatus, y las teorías de la dependencia y el intercambio. Todos ellos son probabilísticos, esto es, consideran que hay una serie de factores de riesgo que aumentan la probabilidad de ocurrencia de esta violencia y, aunque difieren en el modo como lo conceptualizan, todos ellos (excepto el primero) enfatizan el rol del patriarcado en su génesis.

De las diferentes propuestas mencionadas, el modelo ecológico de Heise (1998) constituye uno de los modelos más reconocidos y utilizados, tanto en los diferentes documentos de análisis internacionales (ONU, OMS, UNIFEM, etc.), como en la investigación científica desarrollada en el ámbito de la salud (Carmen VIVES-CASES, 2011). Este modelo, que también es probabilístico, incorpora los principales hallazgos de la investigación antropológica, psicológica y sociológica, y señala como determinantes de la violencia contra las mujeres en la pareja los factores que actúan en el marco sociocultural o macrosistema (como serían, por ejemplo, la aceptación de la violencia como forma legítima de resolver conflictos, la masculinidad vinculada a la dominación, o unos roles rígidos basados en el sexo), los factores que actúan en el marco comunitario o exosistema (como serían, por ejemplo, la pobreza, o el aislamiento de las mujeres y la familia), los factores que actúan en el marco familiar o microsistema (como serían, por ejemplo, los conflictos de pareja, o el control masculino de los bienes), y los factores que actúan en el ámbito individual o historia personal (como serían, por ejemplo, haber presenciado episodios de violencia de pareja en la infancia o el abuso de alcohol u otras sustancias). 
A los modelos multicausales se han añadido posteriormente otras propuestas, como la de Fernando García Seigas y Elena Casado (2010), que ponen el foco explicativo sobre el vínculo afectivo, analizado en términos de reconocimiento y dependencia.

Partiendo del marco interpretativo que proporcionan las investigaciones realizadas desde los estudios feministas y de género (Ana DE MIGUEL, 2008), y en la línea de desarrollar teorías complejas y multivariadas, que, además del patriarcado, incluyeran otros elementos explicativos, hemos propuesto un modelo explicativo cuya presentación constituye el objetivo de este trabajo. Este modelo, al que hemos denominado Modelo Piramidal, ha ido surgiendo a partir de nuestras revisiones de la literatura científica y de las investigaciones previas sobre la cuestión, pero también del contacto directo con mujeres que han padecido esta forma de violencia, y de los resultados de nuestras propias investigaciones (BOSCH; FERRER, 2002; BOSCH; FERRER; Virginia FERREIRO; Capilla NAVARRO, 2013; BOSCH; FERRER; E. GARCÍA; María Carmen RAMIS; C. MAS; NAVARRO; Gema TORRENS, 2008). Como fruto de la reflexión sobre la información aportada por todas estas fuentes, generamos una propuesta de modelo hermenéutico - heurístico que consideramos teóricamente plausible para explicar la violencia contra las mujeres en general, y en la pareja en particular, y en el que, además, encajan los resultados de las investigaciones sobre la cuestión que van conociéndose. Dicho modelo pretende ser sencillo (que no simple), y, a la vez, aplicable a la violencia contra las mujeres en sus diferentes formas, y está centrado en factores explicativos clave, que ordena y jerarquiza. Al tiempo, trata, además, de profundizar en los mecanismos que diferencian a agresores de no agresores.

Cabe puntualizar, sin embargo, que, a pesar de su vocación de aplicabilidad a todas las formas de violencia y a los diferentes contextos (geográficos y sociales), ha sido desarrollado en un entorno europeo y tomando como referencia central la violencia contra las mujeres en la pareja, si bien en su formulación se tuvieron en consideración estudios de muy diversa procedencia (HEISE; Claudia GARCÍA-MORENO, 2003; ONU, 2006).

\section{El modelo piramidal: La escalada hacia la violencia}

El modelo que se propone tiene una estructura piramidal (cuya representación gráfica se presenta en la Figura 1) y consta de cinco escalones. Cuatro de ellos constituyen los mecanismos explicativos de la violencia (sustrato patriarcal, procesos de socialización, expectativas de control, y eventos desencadenantes); y el quinto sería, propiamente, el estallido de la violencia contra las mujeres, en cualquiera de sus diferentes formas.

Figura 1 - Representación gráfica del modelo piramidal.

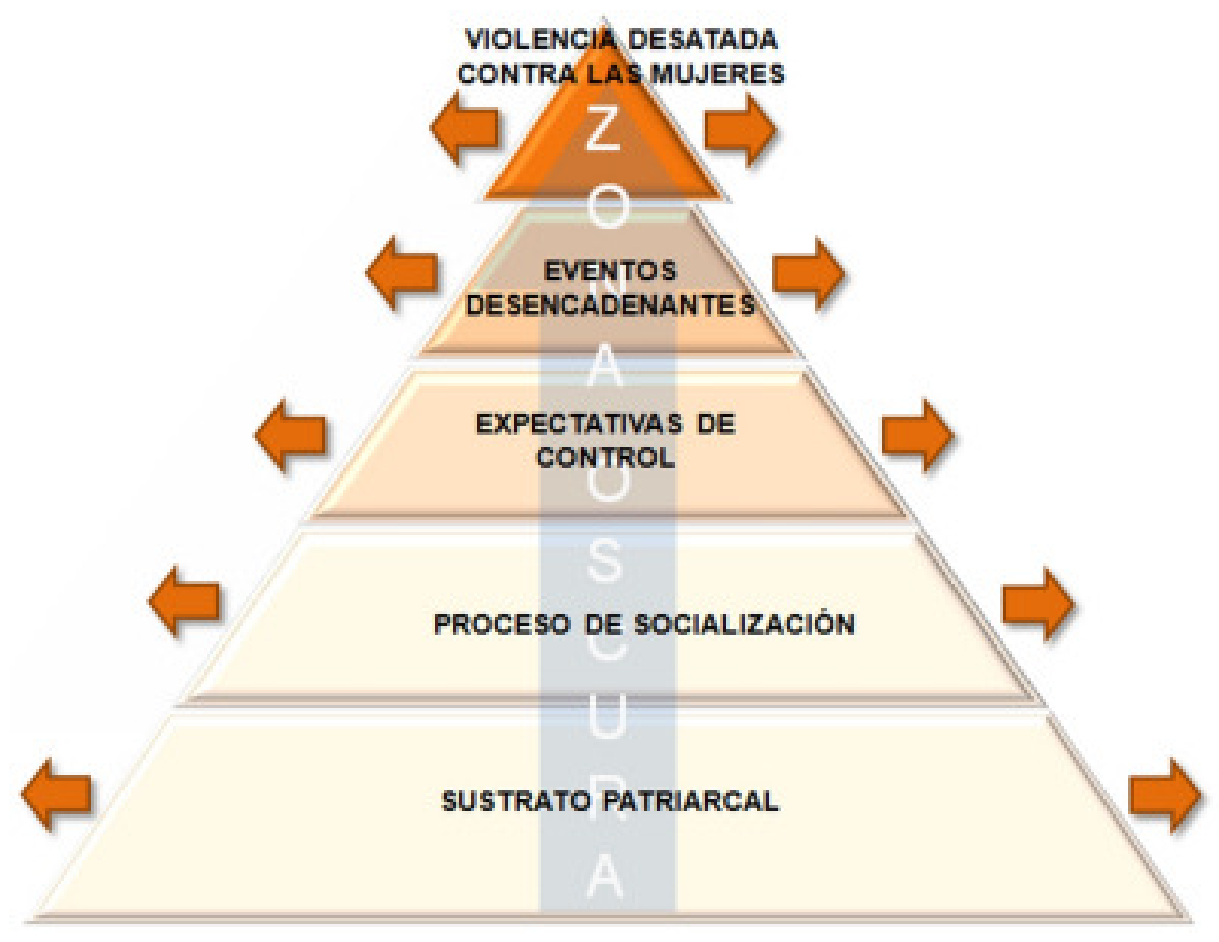

Nota: Imagen elaborada por Virginia Ferreiro Basurto, técnica del grupo de investigación Estudios de Género de la UIB. 


\section{Primer escalón. El sustrato patriarcal}

El Modelo Piramidal propone que la ideología patriarcal constituiría el primer nivel, el sustrato sobre el que se asentaría y desarrollaría la violencia contra las mujeres. Esta ideología tendría como componentes básicos los siguientes:

- El sexismo (actitud hacia una persona por el hecho de que ésta sea hombre o mujer), que puede adoptar diversas formas, desde la misoginia, como máxima expresión del sexismo hostil, hasta el sexismo benévolo, moderno, o neosexismo.

Aunque sólo se haga explícito en el sexismo hostil, de facto, todos comparten una misma base: la dominancia masculina, esto es, la consideración de lo masculino como superior y una visión negativa de las mujeres (en comparación con los varones), a las que consideran como seres inferiores, más débiles (física, intelectual y moralmente), con capacidades limitadas, y que necesitan ser dominadas (paternalismo dominante) y/o protegidas (paternalismo protector) (FERRER; BOSCH, 2010).

Desde el modelo propuesto se considera que el sexismo, y la misoginia como su máxima expresión, constituirían la clave explicativa fundamental para la violencia contra las mujeres y un elemento común y característico de los maltratadores.

Cabe remarcar que en la investigación sobre el tema este concepto ha sido reiteradamente relacionado con la violencia contra las mujeres, y existe abundante reflexión teórica y evidencia empírica sobre la aplicabilidad del sexismo en sus diferentes formas al análisis de la violencia contra las mujeres en la pareja (Walter DEKESEREDY, 201 1; EXPÓSITO, 2011; Gwen HUNNICUTT, 2009; MEGÍAS; Mónica ROMERO; Mercedes DURÁN; Miguel MOYA; Gerd BOHNER, 2011 ; MOYA; Soledad DE LEMUS, 2007; MOYA; EXPÓSITO, 2008; O’NEIL; HARWAY, 1997, 1999; Inmaculada VALOR-SEGURA; EXPÓSITO; MOYA; Karelin LÓPEZ, 2014).

- La legitimidad de la desigualdad de género que se refiere a justificar (a partir de la supuesta inferioridad femenina que viene dada por naturaleza) una estructura social (patriarcal) en la que los hombres tienen más poder y privilegios que las mujeres, la subordinación de las mujeres, y las desigualdades entre unos y otras (división sexual del trabajo, roles sociales diferenciados, etc.). En la pareja esto se traduciría en un conjunto de creencias que legitiman el poder y la autoridad de los maridos sobre sus cónyuges.

También en este caso, la evidencia disponible señala que las normas relacionadas con la autoridad de los varones sobre las mujeres constituyen un factor predictor y de riesgo importante para la ocurrencia de violencia contra las mujeres en la pareja (HEISE; Andreas KATSADAM, 2015; OMS, 2014).

- La legitimidad de la violencia contra las mujeres que hace referencia a justificar el uso de la violencia contra quienes no respetan la autoridad masculina (DE MIGUEL, 2010; Kate MILLETT, 1969/1995; Alicia PULEO, 2005; Janet SALTZMAN, 1992). De hecho, de un modo general se ha señalado que "La esencia del código patriarcal es una representación de la masculinidad a través del dominio sobre la mujer" (Inés ALBERDI, Nuria MATAS, 2002, p. 25). En la pareja esto se traduciría en un conjunto de creencias que justificarían la violencia contra aquellas mujeres que no respetan la autoridad de sus maridos.

También en este sentido existe evidencia empírica que señala que el clima de aceptación y las creencias y actitudes tolerantes hacia la violencia contra las mujeres en la pareja constituyen un importante factor predictor y de riesgo sociocultural para su ocurrencia (Robert ARCHER; Nicola GRAHAM-KEVAN, 2003; Mahbobeb FARAMARZI; ESMAILZADEH; MOSAVI, 2005; Michael FLOOD; Bob PEASE, 2008, 2009; Enrique GRACIA; Fernando GARCÍA; Marisol LILA, 2009; GRACIA; Juan HERRERO, 2006; HEISE; KATSADAM, 2015; Helmut KURY; Joachim OBERGFELL-FUCHS; Gunda WOESSNER, 2004; Madhabika B. NAYAK; Christina A. BYRNE; Mutsumi K. MARTín; Anna g. Abraham, 2003; OMS, 2014; OPS, 2013; STITH; Douglas SMITH; Carrie PEN; David WARD; Cari TRITT, 2004; Olalekan A. UTHMAN; Tahereh MORADI; Stephen LAWOKO, 2011).

Igualmente, la evidencia disponible señala que estas actitudes que justifican el uso de la violencia a menudo se caracterizan por minimizar la importancia de la agresión y por justificar o exonerar al perpetrador o culpar a la víctima (VALOR-SEGURA; EXPÓSITO; MOYA, 2011), y que hay diferencias entre mujeres y hombres en cuanto a estas cuestiones (LaVerne BERKEL; Beverly VANDIVER; Angela BAHNER, 2004; FERRER; BOSCH, 2014; FLOOD; PEASE, 2009; NAYAK et al., 2003).

\section{Segundo escalón. La socialización diferencial}

El segundo nivel del Modelo Piramidal (al que hemos denominado procesos de socialización) hace referencia a la difusión de la ideología patriarcal. Concretamente, los constructos básicos relevantes en el marco de este modelo explicativo mediante los cuales se articularía la difusión de dicha ideología durante el proceso de socialización serían los siguientes:

En primer lugar estarían los mandatos de género tradicionales, esto es, las normas de comportamiento que se derivan de la ideología patriarcal, que, desde ella, se consideran como adecuadas y apropiadas para varones y mujeres (en qué consiste ser un hombre masculino y una mujer femenina), y que se caracterizan del modo siguiente (Amparo BONILLA, 2008; Luis BONINO, 
2001 ; Robert CONNELL; James MESSERSCHMIDT, 2013; Enrique DÍEZ, 2015; Marcela LAGARDE, 2005; Isabel MARTÍNEZ-BENLLOCH, 2008; Erik PESCADOR, 2010; M. Angeles REBOLLO, 2010):

- El mandato de género tradicional masculino, se caracterizará como ser-para-sí, asociando la masculinidad con el control, el poder, la dominación, la fuerza, el éxito, la racionalidad, la autoconfianza y la seguridad en uno mismo y las tareas productivas (como el trabajo remunerado - la política, que responsabilizan a los varones de los bienes materiales). En esencia, este mandato incluye no poseer ninguna de las características que se les suponen a las mujeres y contrapesar éstas con sus opuestos (racionalidad por oposición a irracionalidad, fuerza frente a debilidad, ausencia de emociones frente a emocionalidad,...).

- El mandato de género tradicional femenino se caracterizaría como ser-para-otros, asociando la feminidad con la sumisión, la pasividad, la dependencia, la obediencia, la abnegación, la renuncia, y las tareas reproductivas (como el cuidado de la pareja, los/as hijos/as, etc., que responsabiliza a las mujeres del bienestar de los demás y de los bienes emocionales). Vinculado a su rol como como cuidadora y responsable del bienestar de otros/as, este mandato otorga un lugar central a los roles de esposa y madre (hasta considerar que su plenitud y satisfacción sólo puede alcanzarse ejerciendo estos roles, especialmente, a través de la maternidad), y un peso importante a la (supuesta) predisposición al amor, hasta el punto de considerar a las mujeres como completas sólo cuando pertenecen a alguien.

De acuerdo con el Modelo Piramidal propuesto, estos mandatos tendrían su corolario en la denominada ideología de género tradicional, y una fuerte vinculación con modelo de amor imperante.

La ideología de género es el conjunto de creencias que las personas poseemos sobre cuáles son los roles, y comportamientos considerados apropiados para varones y mujeres (por razón de su sexo) y sobre las relaciones que unos y otras deben mantener entre sí (FERRER; BOSCH, 2010; MOYA, 2004), esto es, las creencias prescriptivas sobre los roles de mujeres y hombres. Esta ideología se concibe como una dimensión cuyos extremos pueden etiquetarse como ideología de género tradicional vs. ideología de género feminista - igualitaria (MOYA; EXPÓSITO; José Luis PADILLA, 2006).

La ideología de género tradicional supone, pues, asumir y validar los mandatos de género tradicionales y se caracteriza por enfatizar las diferencias sexuales o biológicas entre varones y mujeres y, consecuentemente, la necesidad de una estricta diferenciación de roles y ámbitos para unas y otros: como consecuencia de considerar a las mujeres como seres débiles y necesitados de protección, se las relega a los roles de esposa, ama de casa y madre (ámbito privado); como consecuencia de considerar a los varones como seres fuertes con autoridad y protectores, se les asignan roles de proveedor, vinculados a la toma de decisiones y la esfera pública (MOYA; EXPÓSITO; PADILLA, 2006).

Así, los mandatos de género tradicionales se plantean como complementarios en el sentido propuesto por Edgar Sampson (1993): la identidad masculina se define como autónoma, independiente y controladora, pero para que ello sea posible, es necesario que haya quien asuma una identidad dependiente y relacionada con el cuidado y el servicio (la identidad femenina). Pero, el mandato de género masculino implica no sólo una hegemonía externa (la dominación de los varones sobre las mujeres), sino una hegemonía interna, esto es, la ascendencia social de unos varones sobre otros, la subordinación de otras formas de masculinidades alternativas a la masculinidad hegemónica tradicional (DÍEZ, 2015).

Por su parte, el modelo de amor romántico, también denominado amor fusional, relación fusionada o vínculo fusional romántico (BOSCH; FERRER; FERREIRO; NAVARRO, 2013; M. LUz ESTEBAN; Ana TAVORA, 2008; GARCÍA SEIGAS; CASADO, 2010; Coral HERRERA, 2011 ; Marie Lise LABOUTÉ, 2010; Aurora LEAL; Rosa A. NIETO, 2007; Tomasa LUENGO; Carmen RODRÍGUEZ-SUMAZA, 2009; TAVORA, 2007) hace referencia a qué significa enamorarse, qué sentimientos se consideran apropiados y cuáles no, cómo debe ser la relación, y qué papel ha de desempeñar el amor en nuestras vidas. Además, incluye una serie de mitos y creencias irracionales al respecto, como, por ejemplo, que el único requisito para alcanzar la felicidad es tener a la otra persona, que cada miembro de la pareja tiene capacidad para satisfacer completamente todas las necesidades del/la otro/a, que existe la media naranja, etc. (Ana BARRÓN; David MARTÍNEZ-Í̃NIGO; Pilar DE PAUL; Carlos YELA, 1999; FERRER; BOSCH; NAVARRO, 2010; YELA, 2000, 2003). Este modelo lo aprendemos, y, en su caso, lo interiorizamos, durante el proceso de socialización.

Pero el amor no es una experiencia neutra sino fuertemente generizada (Florence DENMARK; Vita RABINOWITZ; Jeri SECHZER, 2005; LEAL, 2012; Gabriela SCHÄFER, 2008), de modo que los mandatos de género condicionarían de forma diferencial tanto la elección del objeto de amor, como la centralidad del amor y la pareja en nuestras vidas (central y de sumisión y renuncia para el mandato de género tradicional femenino, y periférico y de dominio para el masculino).

De hecho, desde el análisis feminista propuesto por Anna Jonásdóttir (1993), el amor sería tan relevante que constituiría una de las claves explicativas del patriarcado, a través de lo que ella denominó 'plusvalía emocional'. Así, según su análisis, si el capital es la acumulación de 
trabajo alienado, la autoridad masculina sería la acumulación de amor alienado (cantidad desproporcionadamente grande de cuidados y amor, tanto directamente como a través de los/as hijos/as, de la que los varones se habrían apropiado), y la explotación consistiría no sólo en la apropiación del trabajo doméstico y de cuidado de las mujeres por parte de los varones, sino también de su dedicación emocional a ellos.

\section{Tercer escalón. Las expectativas de control.}

Una de las acepciones para el término expectativa señala que se refiere a la esperanza de realizar o conseguir algo o a la posibilidad razonable de que algo suceda, es decir, a una suposición más o menos realista. El tercer escalón del Modelo Piramidal tiene que ver, precisamente, con las expectativas de control de los varones sobre las mujeres.

Concretamente, se plantea que aquellos varones que asumen como propia y no cuestionan la ideología de género tradicional ni sus fundamentos y que asumen los mandatos de género tradicionales, que dan lugar al modelo de masculinidad y feminidad hegemónicas imperantes en las sociedades patriarcales, creen tener unos derechos (expectativas de control) sobre las mujeres (y sobre su pareja en particular, en el caso del maltratador), que consideran válidos y legítimos y se comportan en consecuencia, es decir, esperan mantener el control sobre ellas, sobre sus vidas, sus cuerpos, su sexualidad, sus amistades, su economía, etc.

A esto se suma el hecho de que una relación de pareja basada en los mitos del amor romántico, a los que nos hemos referido en el escalón anterior, supone un riesgo añadido de crear falsas expectativas sobre lo que es o ha de ser la pareja (BOSCH; FERRER; FERREIRO; NAVARRO, 2013; BOSCH; FERRER; NAVARRO; FERREIRO; Catalina ESCARRER; RAMIS; Esther GARCÍA, 2012; FERRER et al., 2010).

Por tanto, las expectativas de control serían, en el marco del modelo propuesto, una resultante de las dimensiones anteriores.

\section{Cuarto escalón. Los factores desencadenantes}

Por lo que se refiere a los eventos desencadenantes, se hipotetiza que las expectativas de control se dispararían y/o materializarían ante ciertos eventos desencadenantes, que constituirían el cuarto escalón del Modelo Piramidal. En este contexto, denominamos factores o eventos desencadenantes a aquellos fenómenos o acontecimientos que:

- Pueden ser interpretados como indicios de que pueden perder el control por aquellos varones que asumen como legítimas las expectativas de control sobre las mujeres. Se incluirían aquí circunstancias como que ellas traten de dedicar su atención y su tiempo a otras actividades o personas, que pongan en cuestión su autoridad, su poder o la legitimidad de estos, etc.

- Generan unas circunstancias favorables para exacerbar el control de los varones sobre las mujeres, ensalzando la ideología y/o los mandatos de género tradicionales, aumentando la permisividad hacia el uso de esta violencia, etc. Se incluirían aquí circunstancias como modificaciones legislativas o cambios sociales, por ejemplo.

Así, en el proceso de tránsito a lo largo de las diferentes etapas del Modelo Piramidal, aquellos varones que asumen el mandato de género masculino tradicional (y la ideología patriarcal subyacente), ante un evento (desencadenante) que frustra sus expectativas de mantener un control sobre sus parejas y/o que refuerza (o ellos creen que refuerza) su posición, considerarían legítimo pasar a la acción y poner en práctica estrategias (que incluirían desde los celos hasta la violencia en sus formas más extremas) para recuperarlo o aumentarlo. Algunos trabajos empíricos corroborarían la existencia de una reacción negativa hacia las mujeres (en forma de valoración más negativa y/o de incremento del sexismo) por parte de los varones en situaciones en las que se produce una pérdida de poder (M. Carmen HERRERA; EXPÓSITO; MOYA, 2012) o cuando son las mujeres percibidas como difíciles de controlar (Ana VIDAL; MEGÍAS, 2014).

Los factores o eventos desencadenantes pueden ser de muy diversos tipos y darse tanto a nivel de macrosistema, como de exosistema, microsistema o de historia personal, por emplear la terminología usada en otros modelos ecológicos. En el Modelo Piramidal, y teniendo en cuenta esa mayor o menor cercanía con el individuo, los hemos clasificado en:

- Eventos desencadenantes personales: uso o abuso de alcohol u otras sustancias tóxicas, matrimonio, separación, nacimiento de hijos/as, cambios en la situación laboral, estrés, problemas económicos, demandas de mayor autonomía por parte de la mujer, etc.

- Eventos desencadenantes sociales: situaciones de crisis económica, modificaciones legislativas, cambios de modelo social, etc.

- Eventos desencadenantes político-religiosos: integrismos, gobiernos ultra-conservadores, etc. 
Cabe señalar que la fuerza o potencia de los factores o eventos desencadenantes puede variar de modo sustancial, de forma que algunos de ellos ( $y$, particularmente los de carácter social y políico - religioso) pueden llegar a ser tan intensos que tengan consecuencias no sólo sobre el comportamiento de los varones violentos sino sobre la sociedad en su conjunto (BOSCH; FERRER; FERREIRO; NAVARRO, 2013). Un ejemplo lo encontraríamos en la irrupción del régimen talibán y sus efectos.

Cabe recordar que algunos de estos eventos, y particularmente los de tipo personal, fueron en su momento considerados como causas exclusivas de la violencia de género en los modelos unicausales de corte psiquiátrico (BLÁZQUEZ et al., 2010).

La evidencia actualmente disponible (Heather FORAN; K. Daniel O'LEARY, 2008; Diana GIL; VIVES-CASES; Carlos ÁLVAREZ; Jaime LATOUR, 2006; José SANMARTÍN, 2006; SANMARTíN; Isabel IBARRA; Yolanda GARCÍA; Pilar MARTíNEZ, 2010) muestra, sin embargo, que éstos no constituyen por sí mismos factores causantes de la violencia contra las mujeres, si no, facilitadores o desinhibidores de ésta, e incluso, que su peso puede haberse visto sobredimensionado por un sesgo de publicación (GIL et al., 2006).

También el enamoramiento romántico (en tanto que materialización del modelo de amor romántico) podría constituir uno de esos factores o eventos desencadenantes ya que, como resume LAGARDE (2005): "Al vivir, cada persona trata de realizar el amor ideológicamente aprendido. En la realidad, la mayoría vive frustraciones amorosas porque casi nunca podemos realizar el imaginario amoroso al que estamos vinculados" (p. 361).

Otros trabajos (OPS, 2013; RODRÍGUEZ-MENÉS; SAFRANOFF, 2012; Robert SARTIN; David HANSEN; Matthew HUSS, 2006) han señalado al poder y, particularmente, a la asimetría en el reparto del poder, como uno de los desencadenantes de la violencia contra las mujeres. Es decir, los varones usarían la violencia (física, psicológica y/o sexual) como estrategia para perpetuar la supremacía y el poder masculino o para corregir las diferencias de poder percibido y restaurar el orden tradicional. En este sentido, autoras como Felicia Pratto y Angela Walker (2004), propusieron el Modelo de Poder Basado en el Género, según el cual las relaciones históricamente desiguales entre varones y mujeres han desembocado en una asimetría de poder entre ellos y ellas, que se constituye en un elemento clave en la relación entre unos y otras, y que se configura a partir de cuatro bases o factores dinámicos de poder que son: el uso de la fuerza o la violencia o la amenaza de usarlas, el control de los recursos, la asimetría en las responsabilidades sociales y la ideología de género. Trabajos como el de Elena Morales-Marente (2007) o el de Megías y Montañés (2012) han incidido en estas cuestiones, realizando un extenso análisis sobre cada una de estas cuatro bases y sus dinámicas, tanto en la sociedad como en la pareja.

Cabe apuntar que, si bien este Modelo de Poder Basado en el Género aporta elementos de análisis relevantes para la comprensión de la violencia contra las mujeres en la pareja, plantea como una de las bases de poder en las que se sustenta el uso de la fuerza, lo que entraña un importante grado de solapamiento con la propia violencia que se pretende estudiar (MEGÍAS; MONTAÑÉS, 2012), y, además, focaliza su análisis en la perspectiva de poder diádico, esto es, en el balance y distribución de poder entre los dos miembros de la pareja, de modo que, a nuestro entender, no incorpora de modo explícito la desigualdad social estructural entre varones y mujeres (y que constituiría un sustrato común a todas las parejas, independientemente de su situación concreta), por lo que resultaría incompleto para el análisis de un problema tan poliédrico como el que nos ocupa.

A modo de resumen, puede decirse que, aunque la lista de posibles eventos desencadenantes es amplia y variada, el elemento clave subyacente a todos ellos desde el punto de vista del Modelo Piramidal es que, o bien desatan el miedo del agresor a perder el control sobre ella (o ellas), o bien lo legitiman para ejercerlo con más fuerza.

\section{El estallido de la violencia}

Por lo que se refiere al estallido de la violencia, desde el Modelo Piramidal se entiende que aquellos varones que hayan recorrido los diferentes niveles sin cuestionarlos llegan al último, y despliegan una serie de estrategias (de violencia psicológica, física, económica, sexual, etc.), dando origen a las importantes cifras de incidencia y prevalencia de violencia contra las mujeres en la pareja que han llevado a los organismos internacionales a considerar que: "Ninguna mujer o niña está completamente libre del riesgo o alcance de esta pandemia mundial (la violencia ejercida contra mujeres y niñas)" (ONU - Mujeres, 2011 , p. 32).

\section{El proceso de filtraje o los puntos de fuga}

Además de los niveles descritos, el Modelo Piramidal incorpora un mecanismo o proceso de filtraje para incorporar al análisis las diferencias entre los varones eligen ejercer la violencia, y quienes no lo hacen, que sería un elemento explicativo importante en este modelo, y también un 
elemento novedoso en relación con otros modelos explicativos disponibles puesto que pretende aportar al modelo el análisis de las diferencias entre agresores y no agresores.

El análisis de estos mecanismos fue abordado de un modo más detallado en un trabajo previo (BOSCH; FERRER, 2013).

A modo de breve resumen, cabe aquí reseñar que, partiendo de las aportaciones de Manuel Castells (1998) sobre la construcción de la identidad cultural, y Rebollo (2010) sobre las actitudes hacia los mandatos de género, en el contexto del Modelo Piramidal se propone que en cada uno de los escalones de la pirámide descritos se daría un proceso, al que hemos denominado filtraje, un mecanismo de fuga, de modo que la mayoría de hombres, aún a pesar de haber nacido en un entorno patriarcal, de haber sido socializados con esas claves de análisis y esas expectativas de control $e$, incluso, de haber vivir alguno o algunos de los elementos desencadenantes descritos, nunca lleguen a ejercer la violencia contra las mujeres en ninguna de sus formas, rompiendo con el mandato patriarcal, escapando de la fuerza central que lleva hacia la violencia. Concretamente, se propone la existencia de tres modalidades de identidad masculina, la identidad legitimadora, que supone asumir a título individual la identidad colectiva, diseñada por las instituciones sociales en una cultural (e implica, en este caso, el ejercicio de la violencia contra las mujeres); la identidad de resistencia, que supone apoyar la individualidad como rechazo a la lógica dominante (y supone, por tanto, rechazar la violencia, pero no oponerse activamente a ella); y la identidad de proyecto, que conlleva una redefinición por parte de la persona de su posición en la cultura dominante a partir de la elaboración de nuevas propuestas que supongan una transformación del contexto (y supone, por tanto, rechazar activamente la violencia contra las mujeres y buscar formas alternativas de definir la identidad masculina).

\section{Conclusiones}

A modo de conclusión, podemos señalar que el modelo piramidal expuesto ha sido formulado con dos objetivos básicos: a) Por una parte, la pretensión de integrar aquellos factores recogidos en diferentes modelos sobre la violencia contra las mujeres, cuya capacidad explicativa ha quedado demostrada empíricamente, tratando de completar ciertos aspectos relativos a la interconexión entre los distintos factores y a las diferencias entre varones agresores y no agresores. De modo resumido, se hipotetiza que, en el proceso de tránsito a lo largo de las diferentes etapas del Modelo Piramidal, aquellos varones que asumen el mandato de género masculino tradicional (y la ideología patriarcal subyacente), ante un evento (desencadenante) que frustra sus expectativas de mantener un control sobre sus parejas y/o que refuerza (o ellos creen que refuerza) su posición, considerarían legítimo pasar a la acción y poner en práctica estrategias (que incluirían desde los celos hasta la violencia en sus formas más extremas) para recuperar, mantener o aumentar ese poder. Y b) Por otra parte, el intento de formular un modelo inclusivo, aplicable a las diferentes formas de violencia contra las mujeres, y que recoja los elementos causales comunes a ellas.

Estos dos objetivos son ciertamente muy ambiciosos y la formulación presentada constituye, por el momento, únicamente una aproximación teórica surgida de la investigación desarrollada hasta el momento (BOSCH; FERRER, 2002; BOSCH; FERRER, FERREIRO, NAVARRO, 2013; BOSCH; FERRER; GARCÍA; RAMIS; MAS; NAVARRO; TORRENS, 2008) y de la reflexión sobre los mismos. Tras esta primera y preliminar formulación, queda por delante el reto de profundizar en los diferentes conceptos teóricos que permiten operativizar cada uno de los escalones del modelo propuesto, así como en los mecanismos que los interconectan entre sí, y de desarrollar aquellos análisis empíricos que puedan ser necesarios para delimitar el potencial explicativo, el nivel de aplicabilidad real que puede llegar a tener el modelo formulado, y su relación con otros modelos preexistentes.

\section{Referencias}

ALBERDI, Inés; MATAS, Nuria. La violencia doméstica en España. Barcelona: Fundación La Caixa, 2002.

ALENCAR-RODRIGUES, Roberta de; CANTERA, Leonor. Violencia de género en la pareja: una revisión teórica. Psico, v. 43, n. 1, p. 116-126, 2012.

APA (American Psychological Association). Resolution On Male Violence Against Women. 1999. Disponible en: http://www.apa.org/about/policy/male-violence.aspx. Acceso en: 22/1 1/2017.

ARCHER, John; GRAHAM-KEVAN, Nicola. Do beliefs about aggression predict physical aggression of partner? Aggressive Behavior, v. 29, p. 41-54, 2003.

BOSCH, Esperanza ; FERRER, Victoria A.. La voz de las invisibles. Las víctimas de un mal amor que mata. Madrid: Editorial Cátedra. Colección Feminismos, 2002. 
BOSCH, Esperanza; FERRER, Victoria A.; FERREIRO, Virginia; NAVARRO, Capilla. Violencia contra las mujeres. El amor como coartada. Barcelona: Antrophos, 2013.

BOSCH, Esperanza; FERRER, Victoria A.; GARCÍA, Esther; RAMIS, María Carmen; MAS, C.; NAVARRO, Catalina; TORRENS, Gema. Del mito del amor romántico a la violencia contra las mujeres en la pareja. Madrid: Instituto de la Mujer, 2008.

BOSCH, Esperanza ; FERRER, Victoria A.. Nuevo modelo explicativo para la violencia contra las mujeres en la pareja: el modelo piramidal y el proceso de filtraje. Asparkía, 24, 54-67, 2013.

BOSCH, Esperanza ; FERRER, Victoria A.. La violencia de género: De cuestión privada a problema social. Intervención Psicosocial, 9(1), 7-19, 2000.

BOSCH, Esperanza; FERRER, Victoria A.; NAVARRO, Capilla; FERREIRO, Virginia; ESCARRER, Catalina; RAMIS, María Carmen; GARCÍA, Esther. Profundizando en el análisis del mito del amor romántico y sus relaciones con la violencia contra las mujeres en la pareja: análisis cualitativo. Madrid: Instituto de la Mujer, 2012.

BARRÓN, Ana; MARTíNEZ-IÑIGO; David, DE PAUL; Pilar; YELA, Carlos. Romantic beliefs and myths in Spain. The Spanish Journal of Psychology, v. 2, n. 1, p. 64-73, 1999.

BERKEL, LaVerne A.; VANDIVER, Beverly J.; BAHNER, Angela D. Gender role attitudes, religion and spirituality as predictors of domestic violence attitudes in white college students. Journal of College Student Development, v. 45, p. 119-133, 2004.

BLÁZQUEZ, Macarena; MORENO, Juan Manuel; GARCÍA BAAMONDE, M. Elena. Revisión teórica del maltrato psicológico en la violencia conyugal. Psicología y Salud, v. 20, n. 1, p. 65-75, 2010.

BONILLA, Amparo. "Género, identidades y violencia". In: MARTÍNEZ-BENLLOCH, Isabel (Dir). Imaginario cultural, construcción de identidades de género y violencia: formación para la igualdad en adolescentes. Madrid: Instituto de la Mujer, 2008. p. 15-34.

BONINO, Luis. "Masculinidad, social y sistema sanitario. El caso de la violencia masculina". In: Seminario sobre mainstreaming de género en las políticas de salud en Europa. Madrid: Instituto de la Mujer, 2001. p. 103-111.

CABRUJA, Teresa. Violencia doméstica: sexo y género en las teorías psicosociales sobre la violencia. Hacia otras propuestas de comprensión e intervención. Intervención Psicosocial, v. 13, n. 2, p. 141 $154,2004$.

CASTELLS, Manuel. "El poder de la identidad". In: CASTELLS, Manuel. La era de la información: economía, sociedad y cultura (Volumen II). Madrid: Alianza, 1998.

CONNELL, Robert, W.; MESSERSCHMIDT, James W. Masculinidade hegemônica: Masculinidade hegemônica: Masculinidade hegemônica: repensando o conceito. Estudos Feministas, v. 21 , n. 1, p. 241-282, 2013.

CORVO, Kenneth; DELARA, Ellen. Towards and integrated theory of relational violence: Is bullying a risk factor for domestic violence? Aggression and Violent Behavior, v. 15, p. 181-190, 2010.

DE MIGUEL, Ana. La violencia contra las mujeres. Tres momentos en la construcción del marco feminista de interpretación. Isegoría. Revista de Filosofía Moral y Política, v. 38, p. 129-137, 2008.

DE MIGUEL, Ana. "A la búsqueda de la claridad conceptual: ¿De qué hablamos cuando hablamos del miedo a las mujeres?" In: FUNDACIÓN ISONOMÍA (Ed.). Miedos, culpas, violencias invisibles y su impacto en la vida de las mujeres: iA vueltas con el amori Castellón: Universidad Jaume I, 2010. p. 148-155.

DEKESEREDY, Walter S. Feminist contributions to understanding woman abuse: Myths, controversies, and realities. Aggression and Violent Behavior, v. 16, n. 4, p. 297-302, 2011.

DELGADO, Carmen. "Aspectos cognitivos y emocionales de la violencia de género". In: FIGUERUELO, Angela; DEL POZO, Marta; LEÓN, Marta (Dirs.). Violencia de género e igualdad. Una cuestión de derechos humanos. Granada: Comares, 2013. p. 65-79.

DENMARK, Florence L.; RABINOWITZ, Vita C.; SECHZER, Jeri A. Engendered Psychology. Women and Gender Revisited. Boston: Pearson, 2005 
DÍEZ, Enrique J. (2015). Códigos de masculinidad hegemónica en educación. Revista Iberoamericana de Educación, v. 68, p. 79-98, 2015.

ECHEBURÚA, Enrique; FERNÁNDEZ-MONTALVO, Javier. Tratamiento cognitivo-conductual de hombres violentos en el hogar: un estudio piloto. Análisis y Modificación de Conducta, v. 23, p. 355-384, 1997.

ESTEBAN, M. Luz; TAVORA, Ana. El amor romántico y la subordinación social de las mujeres: revisiones y propuestas. Anuario de Psicología, v. 39, n. 1, p. 59-73, 2008.

EXPÓSITO, Francisca. Violencia de género. Mente y Cerebro, v. 48, p. 20-25, 2011.

EXPÓSITO, Francisca; MOYA, Miguel C. "Violencia de género”. In: EXPÓSITO, Francisca; MOYA, Miguel C. (Coords.). Aplicando la psicología social. Madrid: Pirámide, 2005. p. 201-227.

FARAMARZI, Mahbobeh; ESMAILZADEH, S.; MOSAVI, S. (2005). A comparison of abused and non abused women's definitions of domestic violence and attitudes to acceptance of male dominance. European Journal of Obstetrics \& Gynecology and Reproductive Biology, v. 122, p. 225-231, 2005.

FERNÁNDEZ VILLANUEVA, Concepción. Violencia contra las mujeres: una visión estructural. Intervención Psicosocial, v. 13, n. 2, p. 155-164, 2004.

FERRER, Victoria A.; BOSCH, Esperanza; NAVARRO, Capilla. Los mitos románticos en España. Boletín de Psicología, v. 99, p. 7-31, 2010.

FERRER, Victoria A.; BOSCH, Esperanza. "Detección y comprensión de actitudes sexistas: Importancia de la ideología de género". In: DE LEMUS, Soledad; RYAN, Estrella (Eds.), Coeducación. Propuestas para alcanzar la igualdad de género desde las aulas. Granada: Universidad de Granada, 2010. p. 41-67.

FERRER, Victoria A.; BOSCH, Esperanza. "El papel de la misoginia en la violencia contra las mujeres: nuevas propuestas explicativas”. In: QUILES, M. Nieves; MORALES, J. Francisco; FERNÁNDEZ, Saulo; MORERA, M. Dolores (Eds.). Psicología de la maldad. Cómo todos podemos ser Caín. Madrid: Grupo 5, 2014. p. 123-162.

FLOOD, Michael; PEASE, Bob. Rethinking the significance of attitudes in preventing men's violence against women. Australian Journal of Social Issues, v. 43, p. 547-561, 2008.

FLOOD, Michael; PEASE, Bob. Factors influencing attitudes to violence against women. Trauma, Violence y Abuse, v. 10, n. 2, p. 125-142, 2009.

FORAN, Heahter M.; O'LEARY, K. Daniel. Alcohol and intimate partner violence: a meta - analytic review. Clinical Psychology Review, v. 28, p. 1222-1234, 2008.

GARCÍA SEIGAS, Fernando J.; CASADO, Elena. Violencia en la pareja: género y vínculo. Madrid: Talasa Ediciones, 2010.

GIL, Diana; VIVES-CASES, Carmen; ALVÁREZ, Carlos; LATOUR, Jaime. Alcohol and intimate partner violence: do we have enough information to act? European Journal of Public Health, v. 16, n. 3, p. 279-285, 2006.

GRACIA, Enrique; GARCIA, Fernando; LILA, Marisol. Public responses to intimate partner violence against women: the influence of perceived severity and personal responsibility. The Spanish Journal of Psychology, v. 12, n. 2, p. 648-656, 2009.

GRACIA, Enrique; HERRERO, Juan. Acceptability of domestic violence against women in the European Union: a multilevel analysis. Journal of Epidemiological and Community Health, v. 60, p. 123-129, 2006.

HARWAY, Michele. Intimate Partner Abuse and Relationship Violence. Washington: APA, 2002. Disponible en: http://www.apa.org/about/division/activities/partner-abuse.pdf. Acceso en: 22/11/ 2017.

HEISE, Lori L. "La violencia contra la mujer. Organización global para el cambio". In: EDLESON, Jeffrey L.; EISIKOVITS, Zvi C. (Eds.). Violencia doméstica: La mujer golpeada y la familia. Barcelona: Granica, 1997. p. 19-58. 
HEISE, Lori L.. Violence against women: an integrated ecological framework. Violence Against Women, v. 4 , p. 262-290, 1998.

HEISE, Lori L.; GARCÍA-MORENO, Claudia. "La violencia en la pareja”. In: KRUG, Etienne G.; DAHLBERG, Linda L.; MERCY, James A.; ZWI, Anthony B.; LOZANO, Rafael (Eds.). Informe Mundial sobre Violencia y Salud. Washington, DC: Organización Panamericana de la Salud, 2003. p. 95-132.

HEISE, Lori, L.; KATSADAM, Andreas. Cross-national and multilevel correlates of partnet violence: an analysis od data from population-based surveys. Lancet Global Health, v. 3, p. e332-e340, 2015.

HERRERA, Coral. La construcción sociocultural del amor romántico. Madrid: Fundamentos, 2011.

HERRERA, M. Carmen; EXPÓSITO, Francisca; MOYA, Miguel C. Negative reaction of men to the loss of power in gender relations. The European Journal of Psychology Applied to the Legal Context, v. 4, n. 1, p. 17-42, 2012.

HUNNICUTT, Gwen. Varieties of Patriarchy and Violence Against Women. Resurrecting "Patriarchy" as a Theoretical Tool. Violence Against Women, v. 15, n. 5, p. 553-573, 2009.

JONÁSDÓTTIR, Ana. El poder del amor ¿le importa el sexo a la democracia? Madrid: Cátedra, 1993.

KURY, Helmut; OBERGFELL-FUCHS, Joachim; WOESSNER, Gunda (2004). The extent of family violence in Europe: a comparison of Nacional Surverys. Violence Against Women, v. 10, p. 749-769, 2004.

LABOUTÉ, Marie Lise. Hacia el amor verdadero. Liberarse de la dependencia afectiva. Barcelona: Luciérnaga, 2010.

LAGARDE, Marcela. Para mis socias de la vida. Claves feministas, Barcelona, Horas y Horas, 2005.

LAWSON, Jennifer. Sociological theories of intimate partner violence. Journal of Human Behavior in the Social Environment, v. 22, p. 572-590, 2012.

LEAL, Aurora. Dibujos y textos sobre el amor. Representaciones adolescentes. Madrid: Editorial Académica Española, 2012.

LEAL, Aurora; NIETO, Rosa A. "Características y paradojas de una relación de amor deseable. Un estudio con adolescentes". In: FERRER, Victoria A.; BOSCH, Esperanza (Comps.). Los feminismos como herramientas de cambio social (I): De la violencia contra las mujeres a la construcción del pensamiento feminista. Palma: Universidad de las Islas Baleares, 2007. p. 75-88.

LUENGO, Tomasa; RODRÍGUEZ-SUMAZA, Carmen. El mito de la "fusión romántica" y sus efectos en el vínculo de la pareja. Anuario de Sexología, v. 11, p. 19-26, 2009.

MARTíNEZ-BENLLOCH, Isabel. "Introducción”. In: MARTíNEZ-BENLLOCH, Isabel (Dir).). Imaginario cultural, construcción de identidades de género y violencia: formación para la igualdad en adolescentes. Madrid: Instituto de la Mujer, 2008. p. 8-14.

MEGÍAS, Jesús L.; ROMERO, Mónica; DURÁN, Mercedes; MOYA, Miguel C.; BOHNER, Gerd. Spanish validatiom of the Acceptance of Modern Myths about Sexual Aggression scale (AMMSA). The Spanish Journal of Psychology, v. 14, n. 2, p. 912-925, 2011.

MEGÍAS, Jesús L.; MONTAÑÉS, Pilar. "Prevención primaria de la violencia de género". In: DE LEMUS, Soledad; RYAN, Estrella (Eds.), Coeducación. Propuestas para alcanzar la igualdad de género desde las aulas. Granada: Universidad de Granada, 2010, p. 127-163.

MEGÍAS, Jesús L.; MONTAÑÉS, Pilar. Percepción de las mujeres víctimas de malos tratos sobre la asimetría de poder en la pareja y su relación con la violencia: estudio preliminar. Anales de Psicología, v. 28, n. 2, p. 405-416, 2012.

MILLETT, Kate. Política sexual. Madrid: Cátedra, 1969/1995.

MORALES-MARENTE, Elena. El poder en las relaciones de género. Sevilla: Centro de Estudios Andaluces. Consejería de la Presidencia, 2007.

MOYA, Miguel C. "Actitudes sexistas y nuevas formas de sexismo". In: BARBERÁ, Ester; MARTíNEZ BENLLOCH, M. Isabel (Coords.). Psicología y género. Madrid: Pearson, 2004. p. 271-294. 
MOYA, Miguel C.; EXPÓSITO, Francisca; PADILLA, José Luis. Revisión de las propiedades psicométricas de las versiones larga y reducida de la Escala sobre Ideología de Género. International Journal of Clinical and Health Psychology, v. 6, n. 3, p. 709-727, 2006.

MOYA, Miguel C.; DE LEMUS, Soledad. "Puño de hierro en guante de terciopelo: diferencias de poder, sexismo y violencia de género". In: YUBERO, Santiago; LARRAÑAGA, Elisa; BLANCO, Amalio (Coords.). Convivir con la violencia: un análisis desde la psicología y la educación de la violencia en nuestra sociedad. Cuenca: Servicio de Publicaciones de la Universidad de Castilla La Mancha, 2007. p. 99-116.

MOYA, Miguel C.; EXPÓSITO, Francisca. "Sexismo: los efectos perniciosos de una ideología ambivalente". In: MORALES, J .Francisco; HUICI, Carmen; GÓMEZ, Angel; GAVIRIA, Elena (Coords). Método, teoría e investigación en psicología social. Madrid: Pearson Educación, 2008. p. 537558.

NAYAK, Madhabika B.; BYRNE, Christina A.; MARTÍN, Mutsumi K.; ABRAHAM, Anna G. Attitudes toward violence against women: a croos-nation study. Sex Roles, v. 49, n. 7, p. 333-342, 2003.

O'NEIL, James M.; HARWAY, Michele. A multivariate model explaining men's violence toward women. Violence Against Women, v. 3, n. 2, p. 182-204, 1997.

O'NEIL, James M.; HARWAY, Michele. "Preliminary multivariate model explaining the causes of men's violence against women”. In: HARWAY, Michele; M. O'NEIL, James (Eds.). What causes men's violence against women? Thousand Oaks: Sage, 1999, p. 12-18.

OMS (Organización Mundial de la Salud). Violence against women. Intimate partner and sexual violence against women. Fact sheet $n^{\circ} 239,2014$.

ONU - Mujeres. El progreso de las mujeres en el mundo. 2011-2012. En busca de la justicia. Nueva York, NJ: Autor, 2011 . Disponible en: http://www2.unwomen.org/-/media/field\%20office\%20mexico/ documentos/publicaciones/2012/el\%20progreso\%20de\%20las\%20mujeres\%20en\%20el\%20mun do\%202011-2012/el\%20progreso\%20de\%2Olas\%20mujeres\%20en\%20el\%20mundo\%20comple to\%20pdf.pdf?la=es Acceso en: 22/11/2017.

ONU (Organización de Naciones Unidas). Estudio a fondo sobre todas las formas de violencia contra la mujer (AG 61/122/Add. 1). Nueva York, NJ: Autor, 2006. Disponible en: http://www.ceipaz.org/ images/contenido/Estudio\%20a\%20fondo\%20sobre\%20todas\%20las\%20formas\%20de\%20vio lencia\%20contra\%20la\%20mujer.pdf Acceso en: 22/1 1/2017.

OPS (Organización Panamericana de la Salud). Violencia infligida por la pareja. Serie: Comprender y abordar la violencia contra las mujeres. Washington: Organización Mundial de la Salud, 2013.

PESCADOR, Erik. "Reconstrucción de las masculinidades". In: DE LEMUS, Soledad; RYAN, Estrella (Eds.). Coeducación. Propuestas para alcanzar la igualdad de género desde las aulas. Granada: Universidad de Granada, 2010. p. 101-126. p. 101-126.

PRATTO, Felicia; WALKER, Angela. "The bases of gendered power". In: EAGLY, Alice H.; BEALL, Anne E.; STERNBERG, Robert J. (Eds.). The psychology of gender. New York: The Guilford Press. $2^{\circ}$ edition, 2004. p. 242-268.

PULEO, Alicia. El patriarcado ¿una organización social superada? Temas para el Debate, v. 133, p. 39-42, 2005.

REBOLLO, M. Ángeles. "Perspectivas de género e interculturalidad en la educación para el desarrollo". In: Género en la educación para el desarrollo. Abriendo la mirada a la interculturalidad, pueblos indígenas, soberanía alimentaria, educación para la paz. Madrid: ACNUR: Las Segovias, 2010. p. 11-32.

RODRÍGUEZ-MENÉS, Jorge; SAFRANOFF, Ana. Violence against women in intimate relations: a contrast in five theories. European Journal of Criminology, v. 9, n. 6, p. 584-602, 2012.

SALTZMAN, Janet. Equidad y género: Una teoría integrada de estabilidad y cambio. Madrid: Cátedra. Colección Feminismos, 1992.

SAMPSON, Edgar. Celebrating the other. A dialogical account of human nature. Londres: Harvester Wheatsheaf, 1993. 
SANMARTíN, José. II Informe Internacional Violencia contra la Mujer en las Relaciones de Pareja. Estadísticas y Legis/ación. Valencia: Centro Reina Sofía para el Estudio de la Violencia, 2006.

SANMARTÍN, José; IBARRA, Isabel; GARCÍA, Yolanda; MARTíNEZ, Pilar. III Informe Internacional Violencia contra las Mujeres en las Relaciones de Pareja Estadísticas y Legislación. Valencia: Centro Reina Sofía para el Estudio de la Violencia, 2010.

SARTIN, Robert M.; HANSEN, David J.; HUSS, Matthew T. Domestic violence treatment response and recidivism: a review and implicarions for the study of familia violence. Aggresion and Violent Beahviour, v. 11 , p. 425-440, 2006.

SCHÄFER, Gabriela. Romantic love in heterosexual relationships: women's experiences. Journal of Social Sciences, v. 16, n. 3, p. 187-197, 2008.

STITH, Sandra M.; FARLEY, Sarah C. A predictive model of male spousal violence. Journal of Family Violence, v. 8, n. 2, p. 183-201, 1993.

STITH, Sandra M.; SMITH, Douglas B.; PEN, Carrie E.; WARD, David B.; TRITT, Dari. Intimate partner physical abuse perpetration and victimization risk factors: A meta-analytic review. Aggression and Violent Behavior, v. 10, p. 65-98, 2004.

TAVORA, Ana. "El amor de pareja en la construcción de la identidad de las mujeres". In: Congreso Estatal De las casas de acogida a los Centros de Atención Integral. Madrid: Instituto de la Mujer, 2007. p. 80-94.

UTHMAN, Olalekan A.; MORADI, Tahereh; LAWOKO, Stephen. Are individual and community acceptance and witnessing of intimate partner violence related to its ocurrence? Multilevel structural equation model. PlosOne, v. 6, n. 12, p. 1-8, 2011.

VALOR-SEGURA, Inmaculada; EXPÓSITO, Francisca; MOYA, Miguel C.; LÓPEZ, Karelin. Violencia hacia la mujer en España y Cuba: una misma realidad, dos visiones diferentes. Revista de Psicología Social, v. 29, n. 1, p. 150-179, 2014.

VALOR-SEGURA, Inmaculada; EXPÓSITO, Francisca; MOYA, Miguel C. Victim blaming and exoneration of the perpetrator in domestic violence: the role of beliefs in a just world and ambivalent sexism. The Spanish Journal of Psychology, v. 14, p. 195-206, 2011.

VIDAL, Ana; MEGÍAS, Jesús L. Attributions of blame to battered women whrn they are perceived as feminists or as difficult to deal with. The Spanish Journal of Psychology, v. 17, p. 1-10, 2014.

VIVES-CASES, Carmen. Un modelo ecológico integrado para comprender la violencia contra las mujeres. Feminismo/s, v. 18, p. 291-299, 2011.

YELA, Carlos. El amor desde la psicología social. Ni tan libres ni tan racionales. Madrid: Pirámide, 2000.

YELA, Carlos. La otra cara del amor: mitos, paradojas y problemas. Encuentros en Psicología Social, v. 1, n. 2, p. 263-267, 2003.

Esperanza Bosch-Fiol (esperanza.bosch@uib.es) es Doctora en Psicología, Profesora Titular de Psicología básica, en el Departamento de Psicología en la Facultad de Psicología de la Universidad de las Islas Baleares.

Victoria Aurora Ferrer-Perez (victoria.ferrer@uib.es) es Doctora en Psicología. Catedrática de Psicología Social de Género en el Departamento de Psicología en la Facultad de Psicología de la Universidad de las Islas Baleares. 


\section{COMO CITAR ESTE ARTÍCULO, DE ACUERDO CON LAS NORMAS DE LA REVISTA:}

BOSCH-FIOL, Esperanza; FERRER-PEREZ, Victoria Aurora. "El Modelo Piramidal: alternativa feminista para analizar la violencia contra las mujeres". Revista Estudos Feministas, Florianópolis, v. 27, n. 2 , e54189, 2019.

\section{CONTRIBUCIÓN DE AUTORÍA}

Esperanza Bosch-Fiol - Concepción y elaboración del manuscrito; Revisión y aprobación de la versión final del trabajo.

Victoria Aurora Ferrer-Perez - Concepción y elaboración del manuscrito; Revisión y aprobación de la versión final del trabajo.

\section{FINANCIACIÓN}

No se aplica

\section{CONSENTIMIENTO DE USO DE IMAGEM}

No se aplica

APROBACIÓN DE COMITÉ DE ÉTICA EN INVESTIGACIÓN

No se aplica

\section{CONFLICTO DE INTERESSES}

No existe conflito de intereses

\section{LICENCIA DE USO}

Este artigo está licenciado sob a Licença Creative Commons CC-BY Internacional. Com essa licença você pode compartilhar, adaptar, criar para qualquer fim, desde que atribua a autoria da obra.

\section{HISTÓRICO}

Recibido en $22 / 11 / 2017$

Aprobado en 28/08/2018

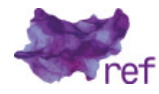

